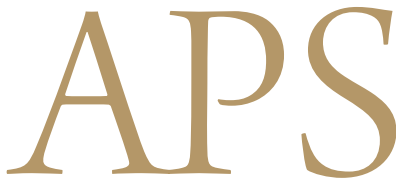

Archives of Plastic Surgery

\title{
The Effect of Phosphatidylcholine and Deoxycholate Compound Injections to the Localized Adipose Tissue: An Experimental Study with a Murine Model
}

\author{
Yongjoon Noh, Chan-Yeong Heo \\ Department of Plastic and Reconstructive Surgery, Seoul National University Bundang Hospital, Seoul National University College of \\ Medicine, Seongnam, Korea
}

Background Phosphatidylcholine (PPC) and deoxycholate (DCA) compound has been recently used for the purpose of partial lipolysis and is valued for its efficacy and lower invasiveness compared to liposuction and dermolipectomy used previously. In this article, the authors discuss the efficacy of the PPC dissolved in DCA via an experimental rat study model, along with suggesting a useful animal experimental model for the study of adipose tissue and lipolysis.

Methods Bilateral inguinal fat pads of an experimental rat were elevated with the deep inferior epigastric vessel as the sole vascular pedicle. Normal saline was injected on one side as a control group and a PPC and DCA compound was injected on the other side. After 4 days, the rats were euthanized for microscopic tissue examination. The pathology was scored by a semiquantitative system in 4 categories: normal fat amount, fat necrosis, inflammatory activity, and stage of fibrosis. A Wilcoxon signed-rank test powered by SPSS packet program was used for statistical analysis and to determine significance.

Results Microscopic examination was performed on the obtained samples, and the experimental data of all four categories showed significant histologic differences compared to the control group. All of the data also showed statistical significance by the Wilcoxon signedrank test $(\mathrm{P}<0.01)$.

Conclusions In the inguinal fat pad rat model, the control group and the experimental group had a differed significantly in the amount of normal fat tissue, inflammation, necrosis, and fibrosis. We recommend the rat inguinal fat pad model used in this study, as it is likely to be useful in related research.

Keywords Phosphatidylcholine / Lipolysis / Fat body / Deoxycholate
Correspondence: Chan-Yeong Heo Department of Plastic and Reconstructive Surgery, Seoul National University Bundang Hospital, Seoul National University College of Medicine, 82 Gumi-ro 173beon-gil, Bundang-gu, Seongnam 463-707, Korea

Tel: +82-31-787-7229

Fax: +82-31-787-4055

E-mail: lionheo@snu.ac.kr

This study was supported by grant 02-2009-021 from the Seoul National University Bundang Hospital Research Fund.

This article was presented as a poster at the 3rd Asian Biomaterials Congress on September 15-17, 2011 in Busan, Korea.

No potential conflict of interest relevant to this article was reported.

Received: 30 Apr 2012 • Revised: 31 Jul 2012 • Accepted: 31 Jul 2012

pISSN: 2234-6163・ elSSN: 2234-6171 • http://dx.doi.org/10.5999/aps.2012.39.5.452 • Arch Plast Surg 2012;39:452-456

\section{INTRODUCTION}

The most prevalent technique for body contouring has been liposuction. Recently, many methods for obesity treatment have been introduced worldwide and many case reports have been published. Phosphatidylcholine (PPC) and deoxycholate

Copyright (๑) 2012 The Korean Society of Plastic and Reconstructive Surgeons

This is an Open Access article distributed under the terms of the Creative Commons Attribution Non-Commercial License (http://creativecommons.org/

licenses/by-nc/3.0/) which permits unrestricted non-commercial use, distribution, and reproduction in any medium, provided the original work is properly cited.

www.e-aps.org 
(DCA) compound is one of them, and was introduced for efficiently reducing fat tissue without serious complications.

PPC is composed primarily of lecithin, which is a main constituent of beans. This substance prevents fatty accumulation and is used to treat liver failure induced by fatty liver, myocardial ischemia, cerebrovascular diseases, and dementia. It has also been recently introduced for lipolysis for obesity treatment in the United States, Europe, and South America. Deoxycholate is used to produce a detergent effect so that the PPC can be welldissolved.

Partial lipolysis performed with PPC and DCA injection is more efficient, effective, and non-invasive technique than the liposuction and dermolipectomy that were previously performed. Some doctors are currently using this technique for treating buffalo hump, lipoma, and xanthelasma related to human immunodeficiency virus and some private clinics are using it for local fat removal of the thighs, flanks, neck, and face [1-7].

Although the interest in PPC is currently increasing, the study of of the lipolysis effect of PPC has not been thoroughly studied. Some studies about lipolysis have been reported using a rabbit model but their results areinconclusive [8-10].

In this article, the authors report a preclinical study on the histologic effect of PPC injection to the adipose tissue in a rat model and establish a new rat model for such research.

\section{METHODS}

Fifteen male Sprague-Dawley rats weighing 280 to 300 g were raised under controlled conditions for one week, and their bilateral inguinal areas were used for the experiment. Flaps were elevated at the bilateral inguinal fat pads of the rats, with one side used as the experimental group and the other as the control group. A $2-\mathrm{cm}$ incision was made at the bilateral inguinal area and the inguinal fat pad was exposed (Fig. 1). Communicating branches between the skin and the fat tissue were all clamped and coagulated. This resulted in an island flap composed of the

\section{Fig. 1. Inguinal fat pad of a rat}

\section{Elevation of pedicled inguinal fat pad of a rat.}

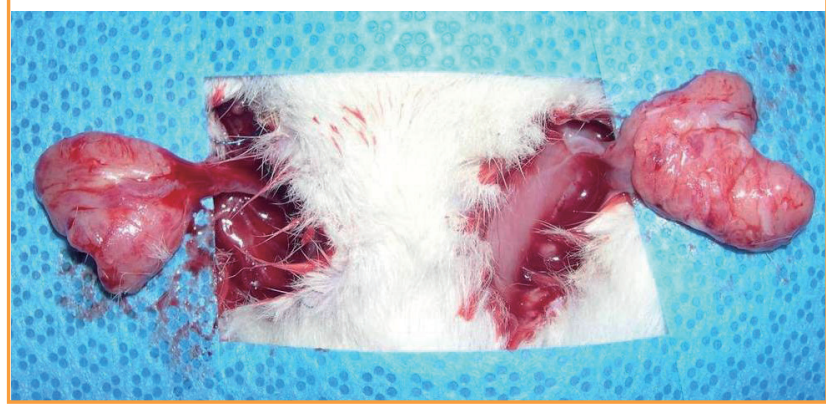

inguinal fat pad with the deep inferior epigastric artery as the only vascular pedicle. Inguinal fat pad anatomy is relatively stationary, ensuring a less complicated dissection in which the environment did not fluctuate in this study. The left inguinal fat pads were used as a control group and $1 \mathrm{~mL}$ saline was injected. On the right side, $0.8 \mathrm{~mL}$ of the PPC/DCA compound and $0.2 \mathrm{~mL}$ of a lidocaine mixture were injected for the experimental group. The components of the PPC solution used in this experiment are described in Table 1. After four days, the rats were euthanized and the tissues were fixed with formalin and embedded into a paraffin block for histologic examination (Fig. 2). Histologic examination was performed by well-trained pathologists in double-blind fashion. The pathologists had no information about which group the sample they were examining was from. Four factors were examined in each sample: the quantity of normal fat, fat necrosis, inflammatory activity, and stage of fibrosis. Each category was scored in semi-quantitative fashion into one of five grades according to the percentage in one microscopic field ( $\times 100)$ : grade $0(0 \%)$, grade $1(<25 \%)$, grade $2(26 \%$ to $50 \%$ ), grade 3 ( $51 \%$ to $75 \%$ ), and grade 4 (76\% to $100 \%)$. Hematoxylin and eosin staining was used in evaluating the inflammatory status and Masson's trichrome staining was helpful in evaluating the necrotic status of the samples. Statistical analysis was powered by the PASW statistics ver. 18.0 (IBM Inc., New York, NY, USA) and the Wilcoxon signed-rank test was utilized for determining significance.

Table 1. The component of phosphatidylcholine (PPC)/deoxycholate solution used in this experiment

\begin{tabular}{|lr|}
\hline Components of PPC solution in $\mathbf{1}$ vial $(\mathbf{5} \mathbf{~} \mathbf{L})^{\text {a) }}$ \\
\hline Polyunsaturated phosphatidylcholine & $250 \mathrm{mg}$ \\
Sodium deoxycholate & $120 \mathrm{mg}$ \\
Sodium chloride & $12 \mathrm{mg}$ \\
Riboflavin & $0.5 \mathrm{mg}$ \\
Benzyl alcohol & $45 \mathrm{mg}$ \\
\hline a)Produced by Samchundang Pharmaceutics Inc.
\end{tabular}

Fig. 2. Gross photo of fat tissue

Gross photo of inguinal fat pads after 4 days of experiments. PPC, phosphatidylcholine.

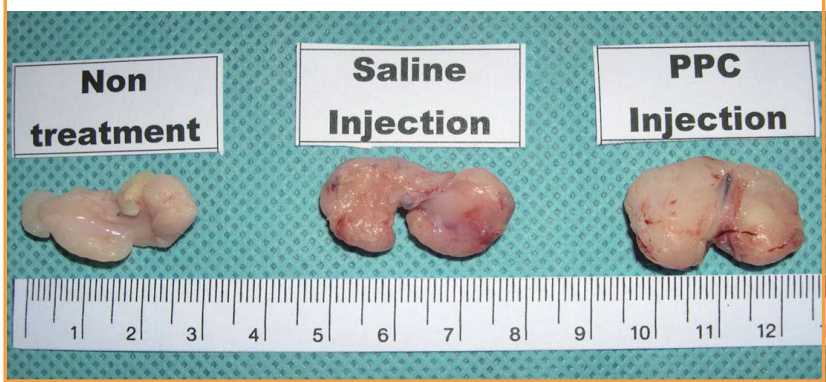




\section{RESULTS}

There was a statistically significant histologic difference between the saline injection group and PPC/DCA injection group (Fig. 3). In the samples from the saline-injected group that were stained with hematoxylin-eosin, normal fat tissue was maintained overall with a mean score of 3.93/4. Also, almost no inflammatory cells were recruited, with a mean score of $0.67 / 4$. In the necrosis analysis, with a mean score of $0.07 / 4$, we could see that saline did not cause the fat cells to necrotize (Fig. 4). On the other hand, the PPC/DCA injected group showed much more inflammatory activity with a mean value of $3.2 / 4$ and a smaller volume of normal fat than the saline-injected group with

\section{Fig. 3. Result of the experiment}

Comparison of normal fat distribution, inflammation, necrosis, and fibrosis between saline-injected fat tissue and phosphatidylcholine (PPC)/deoxycholate injected fat tissue. All categories showed significant differences $(\mathrm{P}<0.01)$.

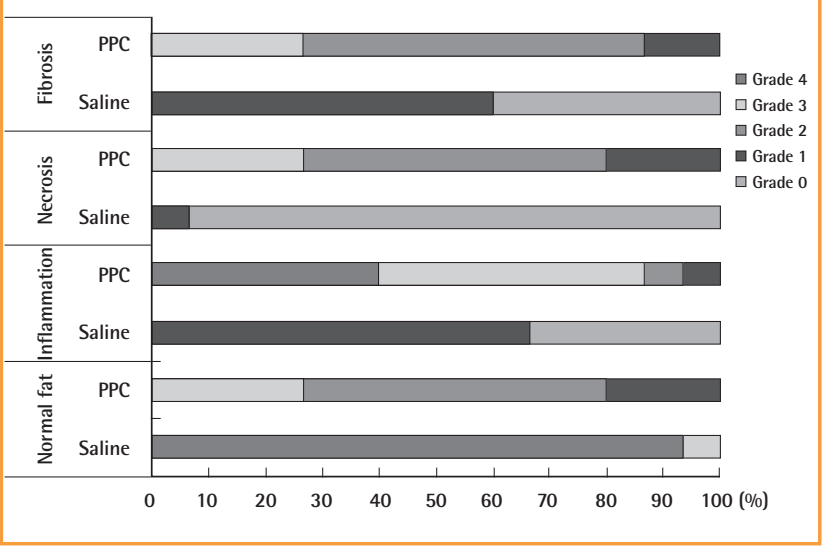

\section{Fig. 4. Microscopic view of saline injected fat tissue}

Microscopic examination of H\&E stained fat tissue, $\times 100$ magnification of saline injected fat tissue.

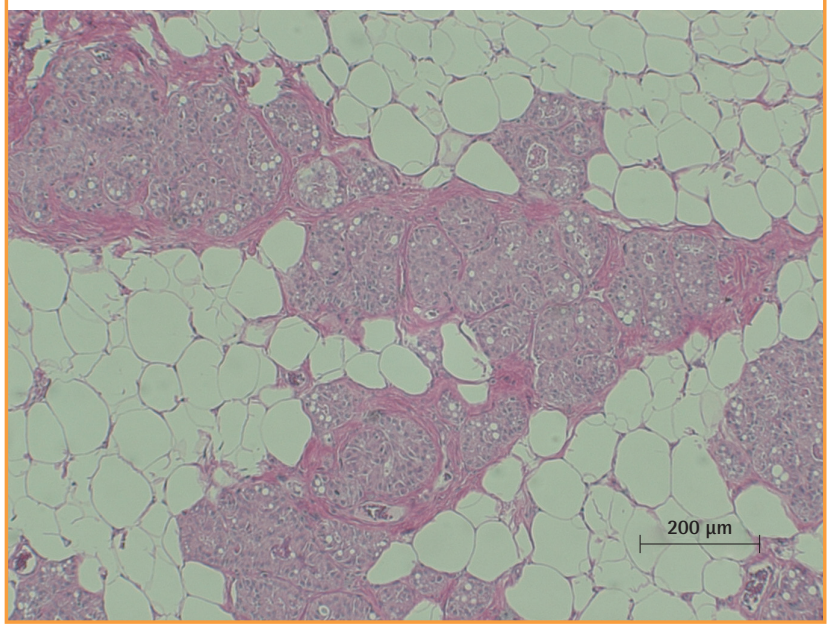

a mean value of 2.07/4. Regarding necrosis, significantly more cells in the experimental group resulted in necrosis than in the saline-injected group, showing a mean value of 2.07/4 (Fig. 5). Fibrosis was examined under Masson's trichrome staining and in the saline-injected group, minimal fibrosis was found with a mean value of 0.6/4 (Fig. 6). In the PPC/DCA-injected group, however, a significant amount of fibrosis was shown, as the mean value jumped up to 2.13/4 (Fig. 7). All of the values showed statistical significance when analyzed by the Wilcoxon signed-rank test following the semi-quantitative scoring system $(\mathrm{P}<0.01)$.

\section{Fig. 5. Microscopic view of PPC/DCA injected fat tissue}

Microscopic examination of H\&E stained fat tissue, $\times 100$ magnification of PPC-injected fat tissue. A histiocytic and giant cell reaction are seen with fat necrosis. Neutrophilic infiltrate is also seen, indicating an inflammatory response. PPC, phosphatidylcholine; DCA, deoxycholate.

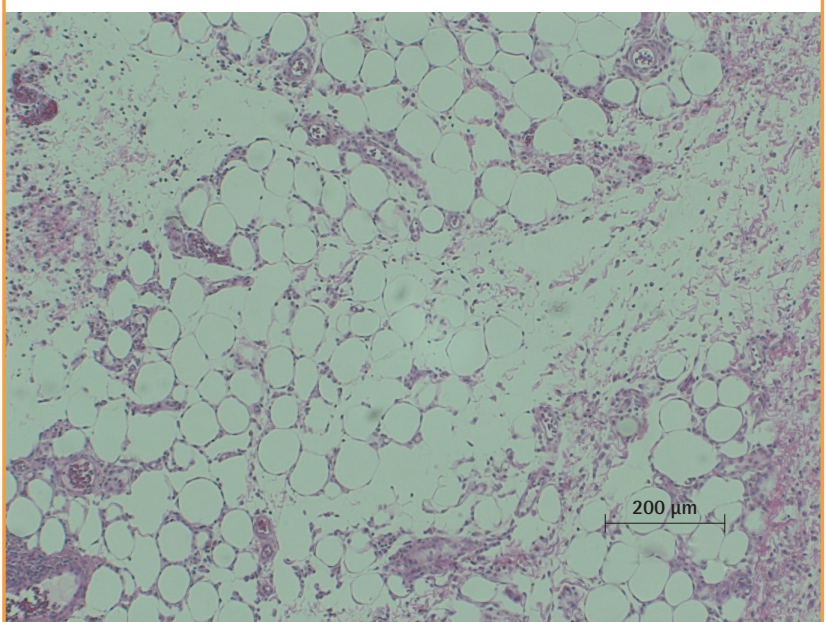

Fig. 6. Microscopic view of saline injected fat tissue

Microscopic examination of Masson's trichrome-stained fat tissue, $\times 100$ magnification of saline-injected fat tissue.

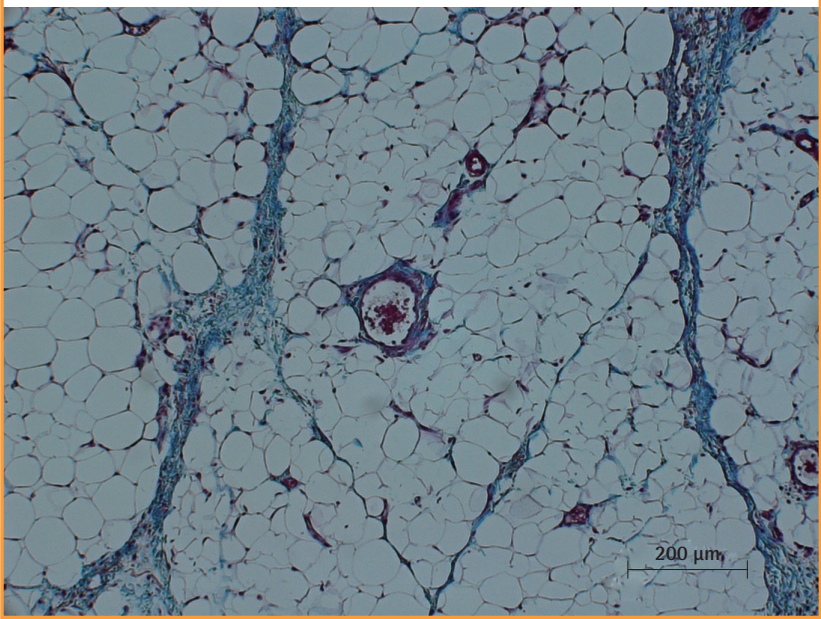




\section{Fig. 7. Microscopic view of PPC/DCA injected fat tissue}

Microscopic examination of Masson's trichrome-stained fat tissue, $\times 100$ magnification of PPC-injected fat tissue. PPC, phosphatidylcholine; DCA, deoxycholate.

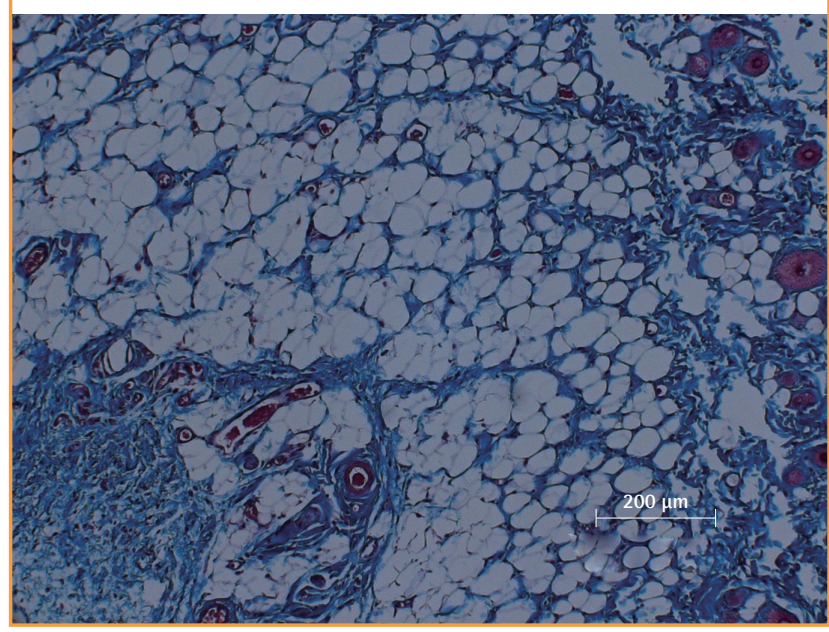

\section{DISCUSSION}

Traditionally accepted treatments for obesity were diet control, exercise, and behavior therapy, and drug usage is also increasing. Obesity is now considered to be a disease and at the same time an important risk factor for hypertension, diabetes mellitus, and cardiovascular diseases. It is obvious that treating obesity may help in prophylaxis of these related diseases and their complications, but this should be clearly distinguished from body contour correction, which reduces the fat tissue of a specific part of the body. Body contour correction can sometimes reduce the risk factors of obesity but more importantly, it increases patients with their body image. A typical example of body contouring is liposuction. It is an invasive procedure that is performed under general anesthesia and is relatively expensive. Moreover, fatal complications may take place, such as fat embolism and sepsis, although the incidence is very rare. For this reason, many new body contouring procedures has been recently introduced for local fat tissue reduction. Although many of these procedures are available to the public, few of them have been properly tested for their efficacy and safety for use in the reduction of adipose cells and tissues.

Lately, a drug composed of PPC, which is extracted from soybean oil, was introduced. PPC is mainly composed of lecithin and is an important phospholipid in the cell membrane. This substance influences triglyceride metabolism including cholesterol, and increases the solubility of cholesterol, which may cause changes in the composition of fat deposits and prevents plaque aggregation of adipose tissue. As a result of this pharmacologic mechanism, PPC is currently used as a medicine for many diseases such as lipid atheroma, hypercholesterolemia, fat embolism, fatty deposits, atherosclerotic plaque, metal disturbances, and liver cirrhosis caused by drugs or alcohol [11].

PPC has gained some popularity in recent several years as a prevalent form of injection lipolysis material and has become popular in the United States, Europe, and South America for this purpose [12]. Mesotherapy is also an injection form of lipolysis which is currently popular, but it differs from PPC in that the former is a "size-reducing" procedure whereas the latter destroys the adipose cells. This is why the brand names for this therapy include the Lipodissolve and fat-away.

PPC was first introduced into medicine in the 1960s. It was firstly used as a treatment option for cardiovascular accident, cerebrovascular diseases, and dementia prophylaxis. It was in the 1980s when PPC usage was initially reported for aesthetic purposes at the International Convention of Mesotherapy in Paris. In the convention, Doctor Sergio Magguiori from Italy first announced the original use of PPC for its lipolytic effect on eyelid xanthelasma. In the mid-1990's, a Brazilian dermatologist, Doctor Patricia Rittes [13], published a PPC usage manual for removing infraorbital fat tissue. This initiated the invention of many new partial lipolysis techniques worldwide, and clinical studies are now in progress in the US under US Food and Drug Administration approval. In 2006, Doctor Franz Hasengschwandtner [14] from Austria published his findings that 441 patients who received PPC injections all experienced a lipolysis effect. He described in his article that this PPC injection therapy is very safe, as all but 6 patients who were enrolled in his study were satisfied with their results. Likewise, PPC injection as lipolysis treatment is becoming a matter of interest worldwide $[13,15,16]$.

The efficacy of PPC is generally accepted, but there have been relatively few good studies about the safety of the treatment, although few complications have been reported. Also, some recent studies suggest that DCA has some effect in lipo-destruction in vitro [17-20]. This remains a question to be addressed in future research.

The present experiment on the lipolytic effect of PPC/DCA in a rat model has provided a useful clinical basis for the efficacy of PPC/DCA and a foundation for a future clinical trial. The rat inguinal fat pad model we proposed for the study of lipolysis may also become a useful tool as it is anatomically orderly and simple to manipulate.

In conclusion, the PPC/DCA-injected group and the control group had significantly different quantities of normal fat tissue, inflammation, necrosis, and fibrosis in a rat inguinal fat pad model. This suggests the injection of PPC/DCA affects the fat tissue component histologically and this can be the clinical basis for ad- 
ditional safety research and for a future clinical trial.

\section{REFERENCES}

1. Hasengschwandtner F. Phosphatidylcholine treatment to induce lipolysis. J Cosmet Dermatol 2005;4:308-13.

2. Rose PT, Morgan M. Histological changes associated with mesotherapy for fat dissolution. J Cosmet Laser Ther 2005; 7:17-9.

3. Rotunda AM, Kolodney MS. Mesotherapy and phosphatidylcholine injections: historical clarification and review. Dermatol Surg 2006;32:465-80.

4. Palmer M, Curran J, Bowler P. Clinical experience and safety using phosphatidylcholine injections for the localized reduction of subcutaneous fat: a multicentre, retrospective UK study. J Cosmet Dermatol 2006;5:218-26.

5. Co AC, Abad-Casintahan MF, Espinoza-Thaebtharm A. Submental fat reduction by mesotherapy using phosphatidylcholine alone vs. phosphatidylcholine and organic silicium: a pilot study. J Cosmet Dermatol 2007;6:250-7.

6. Pereira LH, Sterodimas A. Correction for the iatrogenic form of banana fold and sensuous triangle deformity. Aesthetic Plast Surg 2008;32:923-7.

7. Bechara FG, Skrygan M, Kreuter A, et al. Cytokine mRNA levels in human fat tissue after injection lipolysis with phosphatidylcholine and deoxycholate. Arch Dermatol Res 2008; 300:455-9.

8. Rittes PG, Rittes JC, Carriel Amary MF. Injection of phosphatidylcholine in fat tissue: experimental study of local action in rabbits. Aesthetic Plast Surg 2006;30:474-8.

9. Salles AG, Valler CS, Ferreira MC. Histologic response to injected phosphatidylcholine in fat tissue: experimental study in a new rabbit model. Aesthetic Plast Surg 2006;30:479-84.

10. Treacy PJ, Goldberg DJ. Use of phosphatidylcholine for the correction of lower lid bulging due to prominent fat pads. J Cosmet Laser Ther 2006;8:129-32.

11. Salti G, Ghersetich I, Tantussi F, et al. Phosphatidylcholine and sodium deoxycholate in the treatment of localized fat: a double-blind, randomized study. Dermatol Surg 2008;34: 60-6.

12. Duncan DI, Palmer M. Fat reduction using phosphatidylcholine/sodium deoxycholate injections: standard of practice. Aesthetic Plast Surg 2008;32:858-72.

13. Rittes PG. The use of phosphatidylcholine for correction of localized fat deposits. Aesthetic Plast Surg 2003;27:315-8.

14. Hasengschwandtner F. Injection lipolysis for effective reduction of localized fat in place of minor surgical lipoplasty. Aesthet Surg J 2006;26:125-30.

15. Hexsel D, Serra M, Mazzuco R, et al. Phosphatidylcholine in the treatment of localized fat. J Drugs Dermatol 2003;2:511-8.

16. Bechara FG, Sand M, Hoffmann K, et al. Fat tissue after lipolysis of lipomas: a histopathological and immunohistochemical study. J Cutan Pathol 2007;34:552-7.

17. Yagima Odo ME, Cuce LC, Odo LM, et al. Action of sodium deoxycholate on subcutaneous human tissue: local and systemic effects. Dermatol Surg 2007;33:178-88.

18. Rotunda AM, Ablon G, Kolodney MS. Lipomas treated with subcutaneous deoxycholate injections. J Am Acad Dermatol 2005;53:973-8.

19. Schuller-Petrovic S, Wolkart G, Hofler G, et al. Tissue-toxic effects of phosphatidylcholine/deoxycholate after subcutaneous injection for fat dissolution in rats and a human volunteer. Dermatol Surg 2008;34:529-42.

20. Rha EY, Kang JA, Lee JH, et al. Comparative analysis about the effect of isolated phosphatidylcholine and sodium deoxycholate for the viability of adipocyte. J Korean Soc Plast Reconstr Surg 2010;37:531-4. 